\title{
APLICAÇÃO DE BIOESTIMULANTE NAS CARACTERÍSTICAS AMPELOMÉTRICAS DA INFRUTESCÊNCIA DA VIDEIRA 'TIETA'
}

\author{
MARCO ANTONIO TECCHIO², ERASMO JOSÉ PAIOLI-PIRES ${ }^{3}$, JOÃO DOMINGOS RODRIGUES ${ }^{4}$, CÁSSIA REGINA \\ YURIKO IDE VIEIRA ${ }^{2}$, MAURILO MONTEIRO TERRA ${ }^{3}$, RENATO VASCONCELOS BOTELHO ${ }^{5}$
}

\begin{abstract}
RESUMO - O ensaio foi conduzido em 2003, em vinhedo de 'Tieta'. O objetivo foi avaliar o efeito de bioestimulante nas características dos cachos de uva. Foi aplicado o produto Stimulate ${ }^{\ddagger}$, que contém em sua fórmula $0,09 \mathrm{~g} \mathrm{~L}^{-1}$ de cinetina (citocinina), $0,05 \mathrm{~g} \mathrm{~L}^{-1}$ de ácido giberélico (giberelina) e $0,05 \mathrm{mg} \mathrm{L}^{-1}$ de ácido indolbutírico (auxina). Os tratamentos consistiram na imersão dos cachos, 15 dias após o florescimento, em solução aquosa de 0,5\% do adjuvante Natura'1 Óleo, acrescidos de 5 doses de Stimulate $:$ 0; 28; $56 ; 84$ e $112 \mathrm{ml} \mathrm{L}^{-1}$. Analisaram-se o comprimento, a largura e o peso dos cachos, bagos e engaço e o diâmetro do pedicelo. O delineamento estatístico foi em blocos ao acaso, com cinco repetições. Concluiu-se que a maior massa fresca dos cachos foi obtida em função do aumento do número de bagos fixados na ráquis e da massa do engaço. O Stimulate ${ }^{f}$ associado ao Natura'1 Óleo provocou o aparecimento de manchas marrons nos bagos e depreciando na qualidade, diminuiu o tamanho dos bagos e atrasou a maturação dos frutos.
\end{abstract}

Palavras chave: Vitis, uva, reguladores vegetais.

\section{EFFECT OF PLANT GROWTH REGULATORS APPLICATION ON THE CLUSTER AND BERRY MORPHOLOGICAL CHARACTERISTICS OF 'TIETA' GRAPES}

\begin{abstract}
The trial was carried out in 2003 in a vineyard of 'Tieta'. The objective was to evaluat the effects of plant growth regulator application on the cluster characteristics. The growth regulator used was the Stimulate witch consists in a mix of $0.09 \mathrm{~g} \mathrm{~L}^{-1}$ of kinetin (cytokinin), $0.05 \mathrm{~g} \mathrm{~L}{ }^{-1}$ gibberellic acid (gibberellin) e $0.05 \mathrm{mg} \mathrm{L}^{-1}$ of indolbutiric acid (auxin). The treatments consisted of the cluster dipping, fifteen days after bloom, in an aqueous solution containing $0.5 \%$ of the surfactant Natura' 1 Óleo, added five dosis of Stimulate ${ }^{\mathrm{f}}: 0,28,56,84 \mathrm{e} 112 \mathrm{ml} \mathrm{L}^{-1}$. The width, length and mass of clusters, berries and rachis, and pedicel diameter were evaluated. The experimental design used was completely randomized blocks. The results showed that the mass of the cluster occurred by the increased of the number of berries per cluster and the mass of the rachis. The Stimulate ${ }^{\mathrm{f}}$ associated to the Natura'l Oil produced brown spots on the skins of berries undervaluating its quality, decreased its size and delaied the harvest. Key words: Vitis, grapevine, plant growth regulators.
\end{abstract}

Para a produção de variedades de uva sem sementes, é necessário um suplemento exógeno de reguladores vegetais visando a aumentar o tamanho da ráquis e dos bagos, pois, na frutificação natural, as dimensões de tais características são bastante reduzidas. Dentre os produtos utilizados para tal finalidade, o ácido giberélico é o mais utilizado comercialmente, em doses variáveis, em função da variedade e da localização do vinhedo.

O ácido giberélico $\left(\mathrm{GA}_{3}\right)$ é utilizado em videiras para o aumento de tamanho e fixação de bagos, descompactação de cachos e eliminação de sementes (Pires \& Botelho, 2002). Em experimento realizado por Pereira \& Oliveira (1979) em 'Itália', constatou-se aumento do número de bagos retidos nos cachos com imersão da inflorescência em solução aquosa de $\mathrm{GA}_{3} 5 \mathrm{mg} \mathrm{L}^{-1}$ antes do florescimento, enquanto com a imersão em 5 ou $10 \mathrm{mg} \mathrm{L}^{-1}$ depois do florescimento, houve aumento da massa dos cachos e bagos.

$\mathrm{O}$ efeito do $\mathrm{GA}_{3}$ nos cachos e bagos da cultivar Maria (IAC 514-6) foi avaliado por Kalil et al. (1999). Com a aplicação de $200 \mathrm{mg} \mathrm{L}^{-1}$, aos 14 dias após o florescimento, houve aumento na massa, comprimento e largura dos cachos, e no número, massa, comprimento e largura dos bagos. Pires et al. (1986) obtiveram resultados semelhantes ao utilizarem $20 \mathrm{mg} \mathrm{L}^{-1} \mathrm{de} \mathrm{GA}_{3}$, com o mesmo objetivo, em videira 'A Dona' (IAC 87113).

Razeto \& Espinoza (1990) e Retamales et al. (1995) relataram a importância de se obterem outros produtos para a melhoria no tamanho dos bagos, uma vez que o ácido giberélico tem alguns efeitos indesejáveis, como o aumento do vigor das plantas, com conseqüente redução na fertilidade das gemas, degrana dos cachos pós-colheita e maior suscetibilidade dos frutos às podridões. Trabalhos mais recentes com outros reguladores vegetais, como o thidiazuron (TDZ), forclorfenuron (CPPU) e o quinmerac, mostraram resultados promissores, como a melhoria das características morfológicas dos cachos e bagos de uvas.

O TDZ (N-fenil-N-1,2,3-tidiazol-5-tiuréia) é um regulador vegetal que está sendo usado em cultura de tecidos para induzir brotação in vitro, pertencendo ao grupo da citocinina não-purínica (Mok et al., 1982), sendo dez mil vezes mais potente que a benzilaminopurina (Meyer $\&$ Kernsh, 1986). Botelho et al. (2003a) e Botelho et al. (2003b) verificaram o efeito de $0 ; 2,5 ; 5,0 ; 7,5 ; 10,0 ; 12,5$ e 15,0 $\mathrm{mg} \mathrm{L}^{-1}$ de TDZ aplicado aos 14 dias após o florescimento em uvas 'Vênus' e 'Niagara Rosada'. Verificaram, nas cultivares avaliadas, aumento da massa dos cachos com as doses crescentes de thidiazuron. Em uvas 'Vênus', o TDZ aumentou o número de bagos, devido à maior fixação de flores na ráquis, enquanto, em uvas 'Niagara Rosada' houve aumento da massa, comprimento e largura dos bagos.

A citocinina associada à giberelina também promove melhoria na qualidade dos frutos de videira. Botelho et al. (2003a), em experimento realizado com 'Niagara Rosada' na região de Jundiaí, obtiveram maior massa, comprimento e largura de bagos nos tratamentos com TDZ 10mg $\mathrm{L}^{-1}$ associado ao $\mathrm{GA}_{3} 100 \mathrm{mg} \mathrm{L}^{-1}$. A noroeste do Estado de São Paulo, Botelho et al. (2004) avaliaram na mesma cultivar o efeito do TDZ 10mg $\mathrm{L}^{-1}$ e do $\mathrm{GA}_{3} 35 \mathrm{mg} \mathrm{L}^{-1}$, associados ou não. Concluíram que duas aplicações semanais de TDZ 5mg L-1 associado ou não ao GA 3 , aos 14 dias após a floração, foram efetivas no aumento da massa e dimensões dos bagos.

O CPPU, nome comum do forclorfenuron [N-(2-cloro-4-pridil)$\mathrm{N}$-feniluréia], é um regulador vegetal que também pertence ao grupo das citocininas não-purínicas, atuando no aumento do tamanho dos bagos quando aplicado nos cachos após o pegamento dos frutos (Dokoozlian et al., 1994), e ação localizada devido a sua baixa mobilidade no interior da planta (Intrieri et al., 1993). O CPPU, quando aplicado sozinho ou associado ao $\mathrm{GA}_{3}$, tem mostrado bons resultados. Em aplicações de CPPU associado ao $\mathrm{GA}_{3}$ na cultivar Sultanina, observou-

\footnotetext{
${ }^{1}$ (Trabalho 023/2005). Recebido: 26/01/2005. Aceito para publicação: 16/08/2005.

${ }^{2}$ Pesquisador Científico do Centro de Frutas -IAC - Av. Luiz Pereira dos Santos, Bairro Corrupira - 13214-820 - Jundiaí-SP - tecchio@iac.sp.gov.br.

${ }^{3}$ Pesquisador Científico, Instituto Agronômico de Campinas, Caixa Postal 28. 13001-970 Campinas, SP. Bolsista do CNPq.

${ }^{4}$ Professor Titular. UNESP. Instituto de Biociências. Departamento de Botânica. Botucatu/SP.

${ }^{5}$ Professor Doutor. Departamento de Agronomia. UNICENTRO. Rua Simeão Camargo Varela de Sá 3.85040-080. Guarapuava/PR.
} 
se aumento de $28 \%$ no tamanho dos cachos em comparação às aplicações isoladas de GA (Reynolds et al., 1992). Leão et al. (1999) estudaram os efeitos do CPPU e GA 3 nos frutos de 'Perlette'. Os melhores resultados em tamanho de frutos foram obtidos com duas aplicações de CPPU 5 ou $10 \mathrm{mg} \mathrm{L}^{-1} \mathrm{em}$ bagos entre $7 \mathrm{e} 9 \mathrm{~mm}$ de diâmetro, sendo a segunda aplicação associada a $\mathrm{GA}_{3} 10 \mathrm{mg} \mathrm{L}^{-1}$. O CPPU retardou a colheita, em média, por oito dias e aumentou o peso da matéria seca dos engaços.

Mervet et al. (2001) obtiveram na cv. Sultanina melhores resultados com a aplicação de CPPU a $5 \mathrm{mg} \mathrm{L}^{-1}$, aplicados em bagos de $6 \mathrm{~mm}$ de diâmetro, em combinação com GA $40 \mathrm{mg} \mathrm{L}^{-1}$. Houve incremento na produtividade total por planta devido ao aumento em peso do engaço, dos cachos e dos bagos; no tamanho em comprimento, largura e compactação dos cachos; ocorreu decréscimo da porcentagem de bagos rachados, seguido de um atraso no amadurecimento, pela redução dos sólidos solúveis totais e pelo aumento da acidez total titulável.

Feitosa (2002) avaliou os efeitos da aplicação do CPPU a diferentes concentrações em aplicações isoladas ou combinados com $\mathrm{GA}_{3}$, no diâmetro dos bagos, no peso dos cachos, bem como na composição química dos frutos da cv. Itália. O melhor resultado auferido foi conseguido pela aplicação de CPPU $10 \mathrm{mg} \mathrm{L}^{-1}$, resultando num incremento de $13,6 \%$ no diâmetro e $32 \%$ no peso dos bagos, respectivamente, comparado à testemunha ou $\mathrm{GA}_{3} 20 \mathrm{mgL}^{-1}$. O CPPU associado ao $\mathrm{GA}_{3}$ retardou a colheita em 8 dias.

O quinmerac ou IUPAC (ácido 7-cloro-3-metilquinolina-8carboxílico) apresenta um modo de ação semelhante ao da auxina, promovendo o alongamento e o aumento no volume celular, tendo como resultado frutos maiores e mais pesados (Pires, 1998).

Miele et al. (2000) avaliaram, na frutificação da videira 'Itália', o efeito de aplicações de quinmerac $0 ; 10 ; 20$ ou $40 \mathrm{mg} \mathrm{L}^{-1}$, cloreto de mepiquat $200 \mathrm{mg} \mathrm{L}^{-1}$, CPPU $5 \mathrm{mg} \mathrm{L}^{-1}$ ou GA3 40 $\mathrm{mg} \mathrm{L}^{-1}$. Em um segundo experimento, as plantas foram aspergidas com CPPU $0 ; 3 ; 4 ; 5 ; 6 ; 8$ ou 12 $\mathrm{mg} \mathrm{L}^{-1}$. O CPPU e GA 3 aumentaram o tamanho dos bagos, não ocorrendo o mesmo efeito positivo com o quinmerac e cloreto de mepiquat. Houve também um aumento linear no peso dos bagos com a adição de CPPU.

Notam-se bons resultados com a mistura de reguladores vegetais nas características físicas dos cachos e bagos de videira. A utilização de produtos comerciais que contenham a mistura auxina, ácido giberélico e citocinina, poderia facilitar o emprego dessa técnica, tornando-a mais acessível ao produtor. Dentre as opções disponíveis atualmente no mercado, o Stimulate ${ }^{f}$ é um bioestimulante com potencial para ser utilizado, pois na composição desse produto tem-se $0,09 \mathrm{~g} \mathrm{~L}^{-1}$ de cinetina (citocinina), $0,05 \mathrm{~g} \mathrm{~L}^{-1}$ deácido giberélico (giberelina) e $0,05 \mathrm{mg}$ $\mathrm{L}^{-1}$ de ácido indolbutírico (auxina). De acordo com Castro et al. (1998), esse produto atua no crescimento e no desenvolvimento vegetal, estimulando a divisão celular, a diferenciação e o alongamento das células, com aumento também da absorção e da utilização dos nutrientes, sendo especialmente eficiente quando aplicado com fertilizantes foliares.

Pouquíssimos são os estudos sobre esse bioestimulante na área de fruticultura, não havendo relatos, particularmente em viticultura. $\mathrm{Na}$ avaliação do efeito de aplicações de Stimulate ${ }^{\ddagger}$ e do fertilizante foliar Micro-Citrus no número de ramos, comprimento dos ramos, número e caracterização de frutos na colheita da laranjeira, Castro et al. (1998) constataram aumento do número de ramos e do peso médio dos frutos por árvore com aplicação isolada de Stimulate ${ }^{f}$ na dose $1 \mathrm{~L} \mathrm{ha}^{-1}$.

Diante do exposto, neste experimento, objetivou-se avaliar os efeitos da aplicação de Stimulate ${ }^{\ddagger}$ nas características ampelométricas dos cachos da videira 'Tieta', variedade que pode ser uma boa alternativa para a diversificação de cultivares em viticultura familiar, posto ser uva rústica, com fruto aspermo, sem semente e com maior valorização no mercado.

Realizou-se o experimento em vinhedo com nove anos de idade, localizado no Bairro do Poste, no município de Jundiaí-SP, situado a $23^{\circ} 06^{\prime} \mathrm{S}$ e $46^{\circ} 55^{\prime} \mathrm{O}$ e $715 \mathrm{~m}$ de altitude, em Argissolo Vermelho-Amarelo. Utilizou-se da variedade 'Tieta' (Suffolk Red Seedless), enxertada sobre o porta-enxerto IAC 766 , no espaçamento de 2,0 x 1,5m e conduzida no sistema de espaldeira.

As podas foram realizadas em 09-08-03. Os tratamentos foram realizados no dia 22-10-03, aos 15 dias após o pleno florescimento, pela imersão dos cachos, durante 15 segundos, em recipiente plástico contendo solução aquosa de $0,5 \%$ do adjuvante Natura'l Óleo, acrescidos de 5 doses de Stimulate ${ }^{f}$ : $0 ; 28 ; 56 ; 84$ e $112 \mathrm{ml} \mathrm{L}^{-1}(0 ; 2,8 ; 5,6$; 8,4 e $11,2 \%$ de Stimulate $^{f}$ na solução).

O delineamento experimental foi em blocos casualizados, com cinco repetições e parcelas constituídas por três cachos de plantas distintas. As videiras na mesma linha de plantio constituíram um bloco, sendo as parcelas sorteadas em cada bloco para a aplicação dos tratamentos. Dessa maneira, utilizaram-se cinco linhas de plantio para a distribuição dos blocos. A colheita foi realizada no dia 23-12-03, quando os frutos estavam perfeitamente maduros comercialmente, sendo avaliadas as variáveis: massa fresca dos cachos, bagos e engaço, número de bagos por cacho, comprimento e largura dos cachos, comprimento e largura dos bagos e diâmetro do pedicelo.

Os dados foram analisados pelo programa Sisvar, sendo realizadas a análise de variância e a regressão polinomial. Para os resultados significativos ao nível de $1 \%$ e $5 \%$, utilizaram-se de equações de regressão ajustadas para expressão do comportamento das variáveis com o aumento das doses de Stimulate ${ }^{\hat{a}}$.

Das variáveis analisadas, apenas para o comprimento de bagos, não houve modelo de regressão significativo, sendo ajustados modelos de regressão para as demais.

Os dados referentes à massa fresca do cacho, bagos e engaço estão nas Figuras 1-A e 1-B, ajustando-se o modelo de regressão linear para expressão dessas variáveis. Houve aumento linear nessas variáveis com o aumento da dose de Stimulate ${ }^{f}$. Para o comprimento dos cachos, como pode ser observado na Figura 1-C, também se verificou comportamento semelhante. Em relação à largura dos cachos e, de acordo com a Figura 1-D, a equação quadrática foi significativa. Houve decréscimo com as doses de 28 e $56 \mathrm{ml} \mathrm{L}^{-1}$ de Stimulate ${ }^{f}$, e aumento significativo na largura dos cachos nas maiores doses. Nas Figuras 1-E e 1-F, tem-se respectivamente o efeito do bioestimulante na largura dos bagos e no diâmetro do pedicelo, havendo em ambos os casos decréscimo linear destas variáveis com o aumento das doses de Stimulate $^{f}$

Nas figuras 1-G e 1-H, são apresentados os resultados da análise de regressão para número de bagos e massa fresca média do bago, havendo acréscimo no número de bagos, de 50 para 64 bagos por cacho, e decréscimo em peso unitário do fruto com o aumento da dose de Stimulate ${ }^{f}$. O aumento do número de bagos foi, possivelmente, em função da citocinina ou da auxina, contidas na composição do Stimulate ${ }^{f}$. Pereira \& Oliveira (1979) e Botelho et al. (2003a, 2003b) também relataram aumento na fixação de bagos com o uso de $\mathrm{GA}_{3} \mathrm{e}$ citocinina (TDZ), respectivamente. Dentre as inúmeras variáveis, como a dose empregada, o estado de desenvolvimento da planta, o CPPU pode produzir diferentes efeitos no cacho, como o de favorecer a retenção de flores na ráquis (Nickell, 1986). A citocinina promove retenção de frutos, entretanto tal mecanismo ainda não está elucidado cientificamente totalmente (Hashim, 2002). O Stimulate ${ }^{f}$ promoveu aumento na massa dos engaços; resultado consonante com o de Mervet et al. (2001), que aplicaram CPPU mais GA após a florada, em 'Itália'. O aumento em peso de cacho aconteceu devido ao maior número de bagos por cacho, e não por aumento de tamanho e peso do bago, discordando dos autores citados na introdução, que auferiram resultados positivos com o uso de citocinina associada à giberelina, excetuando Miele et al. (2.000) que também não obtiveram resultados positivos com a auxina quinmerac.

Com o Stimulate ${ }^{f}$, houve atraso no amadurecimento dos frutos, o mesmo observado por Leão et al. (1999), Mervet et al. (2001) e Feitosa (2002), com a utilização de citocinina combinada à giberelina.

Observaram-se algumas manchas marrons nos bagos com a utilização de Stimulate ${ }^{f}$, sendo mais evidente nas doses acima de $56 \mathrm{ml}$ $\mathrm{L}^{-1}$. Possivelmente, o aparecimento dessas manchas esteve relacionado ao óleo vegetal utilizado como adjuvante, fato esse que deverá ser mais 


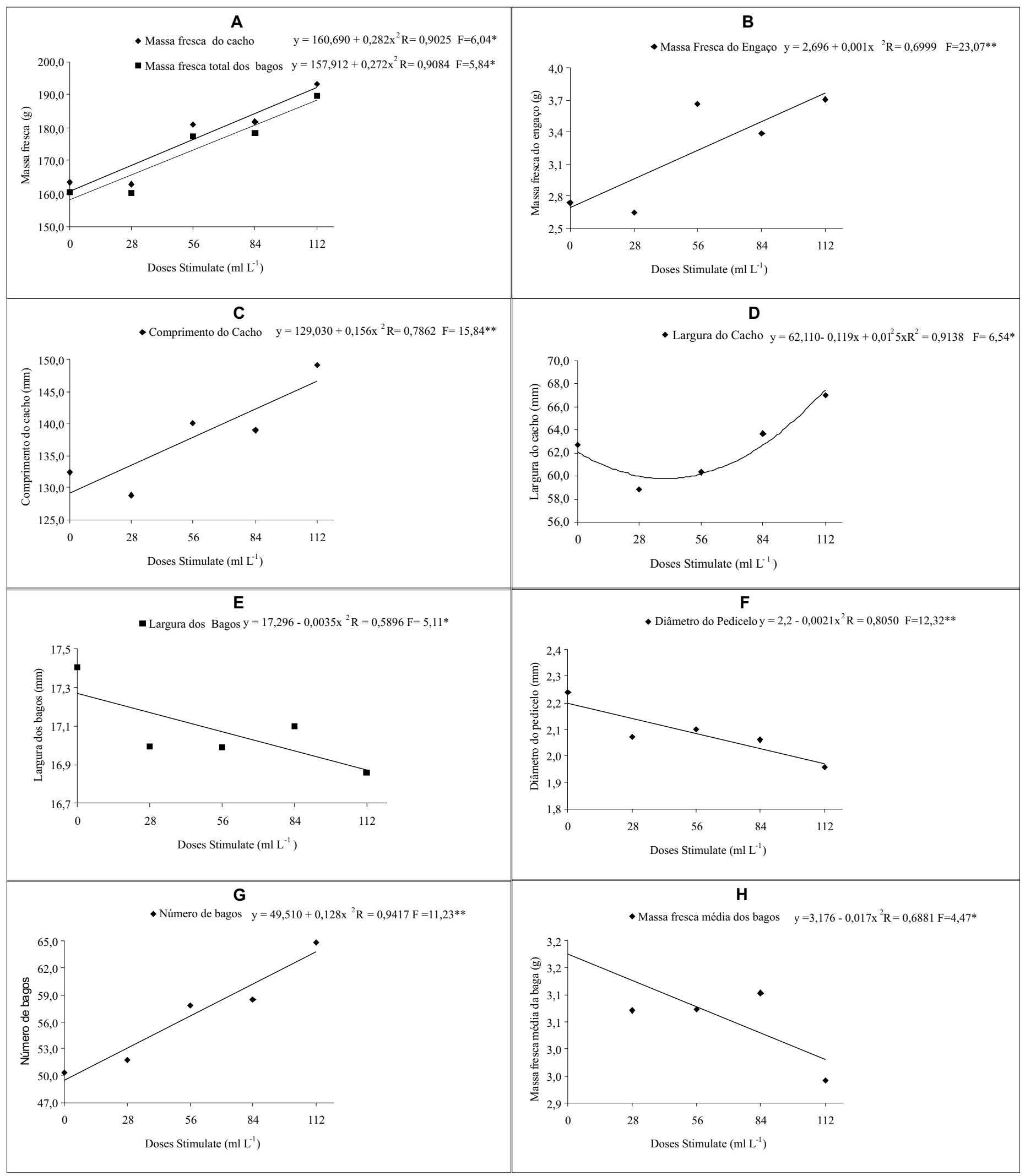

FIGURA 1 - Massa fresca do cacho e bagos (A), massa fresca do engaço (B), comprimento (C) e largura dos cachos (D), largura dos bagos (E), diâmetro do pedicelo $(\mathrm{F})$, número de bagos $(\mathrm{G})$ e massa fresca média do bago $(\mathrm{H})$ de videira 'Tieta, em doses crescentes de Stimulate ${ }^{\mathrm{f}}$, aos 15 dias após o florescimento.

cuidadosamente estudado. Talvez pode ter ocorrido por ter sido feita a imersão dos cachos, pois o óleo vegetal é normalmente recomendado quando se utiliza de pulverização.

A aplicação em doses crescentes de Stimulate ${ }^{f}$ proporcionou aumento na massa do engaço e no número de bagos, diminuindo, no entanto, o tamanho e a massa dos mesmos. Com a dose de $112 \mathrm{ml} \mathrm{L}^{-1}$, obtiveram-se os melhores resultados, havendo também incremento na largura dos cachos. Como efeitos adversos, o Stimulate ${ }^{f}$ proporcionou decréscimo no diâmetro do pedicelo e atraso na maturação. $\mathrm{O}$ aparecimento de manchas marrons nos bagos foi devido, possivelmente, à associação do Stimulate com o Natura'1 Óleo. O presente ensaio não justifica até o momento a utilização desse produto, por ter apresentado 
tais efeitos adversos. No entanto, novos ensaios devem ser realizados visando a um melhor ajuste nas doses adequadas, com a utilização de outro espalhante e/ou modo de aplicação.

\section{REFERÊNCIAS}

BOTELHO, R.V; PIRES, E.J.P.; TERRA, M.M.; CARVALHO, C.R.L. Efeitos do thidiazuron e do ácido giberélico nas características dos cachos e bagas de uvas 'Niagara Rosada' na região de Jundiaí. Revista Brasileira de Fruticultura, Jaboticabal, v. 25, n. 1, p. 96-99, 2003 (a).

BOTELHO, R.V.; PIRES, E.J.P.; TERRA, M.M. Qualidade da uva de mesa 'Vênus' tratada com thidiazuron. Revista Ceres, Viçosa, v.49, n. 286, p. 629-639, 2003b.

BOTELHO, R.M, et al. Efeitos de reguladores vegetais na qualidade de uvas 'Niagara Rosada' na região Noroeste do Estado de São Paulo. Revista Brasileira de Fruticultura, Jaboticabal, v. 26, n. 1, p. 74-77, 2004.

CASTRO, P.R.C.; PACHECO, A.C.; MEDINA, C.L. Efeitos de Stimulate e de microcitros no desenvolvimento vegetativo e na produtividade da laranjeira 'pêra' (Citrus sinensis l. Osbeck). Scientia Agricola, Piracicaba, v.55, n. 2 , p.338-341, 1998.

DOKOOZLIAN, N. K.; MORIYAMA, M.M.; EBISUDA, N. C. Forchlorfenuron (CPPU) increases the berry size and delays the maturity of Thompson Seedless table grapes. In: INTERNACIONAL SYMPOSIUM ON TABLE GRAPES PRODUCTION, 1994, Anaheim, California. Proceedings... Davis: American Society for Enology and Viticulture/University of California, 1994.p. 63-68.

FEITOSA, C.A.M. Efeitos do CPPU e GA no cultivo de uva 'Itália' na região do submédio São Francisco, Nordeste do Brasil. Revista Brasileira de Fruticultura, Jaboticabal, v. 24, n.2, p.348-353, 2002.

HASHIM, J. Gibberellic acid for table grape production. Disponível em: $<$ http:/ucce.ucdavis.edu/counties/cekern/newsletterfiles/ from_the_Vine2702.pdf $>$. Acesso em: abr. 2002.

INTRIERI, C.; FILIPPETTI, I.; PONI, S. Effeti del 'CPPU' sulla crescita delle bache e sulla maturazioni dell'uva in cultivar da tavola apireni e com semi. Rivista di Frutticoltura, Bologna, v.55, n.6, p.57-62, 1993.

KALIL, G.P.C; TERRA, M.M.; KALIL FILHO, A.N.; MACEDO, J.L.V de; PIRES, E.J.P. Anelamento e ácido giberálico na frutificação da uva 'Maria' sem sementes. Scientia Agricola, Piracicaba, v. 56, n. 2 ,p. 317-328, 1999.

LEÃO, P.C.S.; LINO JR., E.C.; SANTOS, E.S. Efeitos do CPPU e ácido giberélico sobre o tamanho de bagas da uva Perlette cultivada no vale do São Francisco. Revista Brasileira de Fruticultura, Jaboticabal, v. 21, n.1 , p. 74-78, 1999.
MERVET, A.K.; ALI, A.; IBRAHIM, H.; RIZK, I.A. Effect of Sitofex (CPPU) on yield and bunch quality of Thompson Seedless grapevines. Egyptian Journal of Agricultural Research, Giza, v.79, n. 2., p. 531-550, 2001.

MEYER, M.M.; KERNSH, R. Thidiazuron and vitro shoot proliferation of Leltius occidentalis L. In: INTERNATIONAL CONGRESS OF PLANT AND CELL CULTURE, 6., 1986, Saint Paul. Abstracts...

MIELE, A; RIZZON, L.A.; DALL'AGNOL, I. Efeito de reguladores de crescimento no tamanho da baga e na composição do mosto da uva 'Italia'. Revista Brasileira de Fruticultura, Jaboticabal, v. 22, n. 2,p. 272-276, 2000.

MOK, M.C.; MOK, D.W.S.; ARMSTRONG, D.J.; SHUDO, K.; ISOGAI, Y.;OKAMOTO, T. Cytokinin activity of N-phenyl-N'-1,2,3-thidiazol5-urea (thidiazuron). Phytochemistry, Oxford, v. 21, p. 1509-1511, 1982.

NICKELL, L.G. Effects of N-(2-chloro-4-pyridyl)-N'-phenylurea on grapes and other crops. Plant Growth Regulation Society of America, v.13, n.2, p.236-241, 1986.

PEREIRA, F.M.; OLIVEIRA, J.C. Efeito da giberilina aplicada antes e depois do florescimento sobre cachos de uva 'Itália'. Científica, Jaboticabal, v.5, n. 2, p. 175-179, 1979.

PIRES, E.J.P. Emprego de reguladores de crescimento em viticultura tropical. Informe Agropecuário, Belo Horizonte, v. 19, n.194, p. 4057, 1998.

PIRES, E.J.P; FAHL, J.I.; CARELLI, M.L.C.; TERRA, M.M.; PASSOS, I.R.S.; CRUZ, L.S.P.; MARTINS. F.P. Respostas à aplicação de ácido giberélico (GA) em panículas de videira do cultivar IAC-871-13 A Dona. In: CONGRESSO BRASILEIRODE FRUTICULTURA, 8., 1986, Brasília. Anais... Brasília: EMBRAPA-DDT/CNPq, 1986. v.3, p.4737.

PIRES, E.J.P.; BOTELHO, R.V. Emprego de Reguladores de crescimento em viticultura. In: Viticultura e Enologia: atualizando conceitos. Belo Horizonte: EPAMIG- FECD, 2002. p.59-81.

RAZETO, B.; ESPINOZA, J. Efecto del ácido giberélico y su forma de aplicacion sobre las yemas u frutos de vide cv. Sultanina. Investigación Agricola, Santiago, v. 10, p. 13-20, 1990.

RETAMALES, J.; BANGERTH, F.; COOPER. T.; CALLEJAS, R; NITO, N.; LOONEY, N.E.; NEVINS D.J.; HALEVY, A.H. Effects of CPPU and $\mathrm{GA}_{3}$ on fruit quality of Sultanina table grape. Acta Horticulturae,.Wageningen, v.26, n. 394, p. 149-157, 1995.

REYNOLDS, A.G.; WARDLE, D.A.; ZUROWSKI,C; LOONEY, N.E. Phenylureas CPPU and thidiazuron affect yield components, fruit composition, and storage potential of four seedless grape selections. Journal of the American Society for Horticultural Science, Alexandria, v.117, n.1, p.85-89, 1992. 